\title{
ON THE CLASS NUMBER OF IMAGINARY QUADRATIC FIELDS ${ }^{1}$
}

\author{
BY A. BAKER
}

\begin{abstract}
A survey is made of our state of knowledge concerning the effective determination of all imaginary quadratic fields with prescribed class numbers. Recent advances are discussed relatıng to linear forms in the logarithms of algebraic numbers fundamental to the theory, and applications are mentioned to other arithmetical questions.
\end{abstract}

It is my privilege once again to address a meeting of the American Mathematical Society, and I am particularly pleased that the present session has been held here in this historic capital. It may perhaps be regarded as an especially appropriate locality, for it was in this city just over half a century ago that Dickson's celebrated History of the theory of numbers was published, and these scholarly tomes have undoubtedly inspired much progress in the topic that forms the theme of my talk. The branch of research about which I shall speak is in fact one of the oldest in the realms of mathematics, originating indeed from the famous Disquisitiones Arithmeticae of Gauss. It was here that Gauss laid the foundations of the theory of binary quadratic forms which, with certain modifications in terminology, has become our modern theory of quadratic fields. Gauss showed how one could divide the set of all binary quadratic forms with a given discriminant into classes such that two forms belong to the same class if and only if there exists an integral unimodular substitution relating them, and he showed also how one could combine the classes into genera so that two forms are in the same genus if and only if they are rationally equivalent. $\mathrm{He}$ also raised a number of notorious problems; in particular, in Article 303, he conjectured that there are only finitely many negative discriminants associated with any given class number and moreover that the tables of discriminants which he had drawn up in the cases of relatively small class numbers were in fact complete. He added, however: "Demonstrationes autem rigorosae harum

AMS 1970 subject classifications. Primary 12A25, 12A50; Secondary $10 \mathrm{~F} 35$.

Key words and phrases. Class number, quadratic fields, Kronecker's limit formula, logarithms of algebraic numbers, genera, generic characters, binary quadratic forms, effective computation, Diophantine equations.

1 An improved text of an address delivered at the 678th meeting of the American Mathematical Society in Washington D. C. on October 31,1970 by invitation of the Committee to Select Hour Speakers for Eastern Sectional Meetings; received by the editors April 12, 1971. 
observationum perdifficiles esse videntur." And apparently Gauss was right, for it was not until 1934 that Heilbronn [14] developing earlier work of Hecke, Mordell and Deuring, succeeded in verifying the first part of the conjecture, and it was only four years ago that Stark [18] and I [3] independently obtained an algorithm for determining all the imaginary quadratic fields with class number 1 , which amounts to a confirmation of the simplest case of the second part of the conjecture. Heilbronn's work was much extended by Siegel [16] and Brauer [10] and the general asymptotic formula for the class number which they established has been of fundamental significance in the evolution of modern number theory; but the arguments are noneffective and cannot lead to a verification of the completeness of the class number tables as sought by Gauss. Stark's work on the class number 1 problem was motivated by an earlier paper of Heegner [13] which related the question to the solution of certain Diophantine equations and the study of elliptic modular functions; my arguments, on the other hand, involved results from the theory of transcendental numbers and an idea of Gel'fond and Linnik [12]. It was shown a year or so ago that the latter method could readily be adapted to give an effective algorithm for determining all the imaginary quadratic fields with class number 2 in the case when the discriminants of the fields are even [5] and a similar algorithm was recently obtained by Kenku in Oxford and Weinberger in Berkeley using analogues of Stark's techniques. Although it was apparent that neither method would extend easily to deal with the case of odd discriminants, it nevertheless became clear ${ }^{2}$ that if one could obtain a basic improvement in the results concerning linear forms in the logarithms of algebraic numbers [3] which formed the key to the transcendental approach to the class number 1 problem then a complete resolution of the class number 2 problem would follow. And some time during this summer Stark (working in Göttingen) and I (in Cambridge) obtained independently a strengthening of the theorem on logarithms of the desired kind [6], [19]. The solutions we derived, though similar in outline, nevertheless differ in some important details. Very recently a combination of our methods has led to improvements in connexion with other applications of linear forms in logarithms [9] and I shall say more about these later. To begin with today I should like to indicate how the theory of transcendental numbers led to a resolution of the class number 1 problem, and I should like then to describe the new ideas involved in solving the analogous problem for class number 2 .

2 This was noted, in particular, by Dr. L. Goldstein of the University of Maryland. 
All solutions to date of the class number 1 problem depend on a formula for a product of $L$-functions analogous to the classical Kronecker limit formula. If $-d<0$ and $k>0$ denote the discriminants of the quadratic fields $Q(\sqrt{ }-d)$ and $Q(\sqrt{ } k)$ respectively and if $\chi(n)=(k / n), \chi^{\prime}(n)=(-d / n)$ denote the usual Kronecker symbols we have

$$
L(1, \chi) L\left(1, \chi \chi^{\prime}\right)=\frac{1}{2} \sum_{f} \sum_{\substack{x=-\infty \\(x, y) \neq(0,0)}}^{\infty} \sum_{\substack{y=-\infty \\ f(x, y)}}^{\infty},
$$

where $f$ runs through a complete set of inequivalent binary quadratic forms with discriminant $-d$. On writing $f=a x^{2}+b x y+c y^{2}$ and assuming that $(k, d)=1$, one can express the sum over $x$ and $y$ as a Fourier series

$$
\frac{\pi^{2}}{3} \frac{\chi(a)}{a} \prod_{p \mid k}\left(1-\frac{1}{p^{2}}\right)+\sum_{r=-\infty}^{\infty} A_{r} e^{\pi i r b /(k a)},
$$

where

$$
\left|A_{r}\right| \leqq \frac{4 \pi}{\sqrt{ } d}|r| e^{-\pi|r| v d /(k a)}
$$

for $r \neq 0$ and

$$
A_{0}=-\frac{4 \pi}{k \sqrt{ } d} \chi(a) \log p
$$

if $k$ is the power of a prime $p, A_{0}=0$ otherwise. Further, assuming that $k>4$, we obtain from classical results of Dirichlet

$$
L(1, \chi)=2 h(k) \log \epsilon / \sqrt{ } k, \quad L\left(1, \chi \chi^{\prime}\right)=h(-k d) \pi / \sqrt{ }(k d),
$$

where $h(k), h(-k d)$ denote the class numbers of the fields $Q(\sqrt{ } k)$ and $Q(\sqrt{ }(-k d))$ respectively and $\epsilon$ denotes the fundamental unit of $Q(\sqrt{ } k)$. Supposing now that $Q(\sqrt{ }-d)$ has class number 1 so that there is just one form $f$ which can be taken as $x^{2}+x y+\frac{1}{4}(1+d) y^{2}$, and choosing $k=21$ so that $h(k)=1, \epsilon=\frac{1}{2}(5+\sqrt{ } 21)$ and $A_{0}=0$, we readily derive the inequality

$$
\left|h(-21 d) \log \epsilon-\frac{32}{21} \pi \sqrt{ } d\right|<e^{-\pi \sqrt{ } d / 100},
$$

provided that $d$ is sufficiently large. But $\pi=-2 i \log i$ and so we have on the left a linear form in two logarithms of algebraic numbers with algebraic coefficients. Gel'fond's work relating to refinements of his solution to Hilbert's seventh problem (see [11]) shows at once that 
this inequality is untenable if $d$ is larger than some effectively computable number and, in principle therefore, all $d$ in question can be found by a finite amount of computation. In practice the bound for $d$ turns out to be about $10^{500}$ and a determination by machine of all solutions of the basic inequality below this figure is quite feasible. However, the computation is superfluous here, for it was proved by Heilbronn and Linfoot [15] in 1934 that, apart from the nine discriminants listed by Gauss, there could be at most one more, and calculations [17] had shown that the tenth $d$, if it existed, would exceed $\exp \left(2 \cdot 2 \times 10^{7}\right)$. The argument I have just given is similar to that indicated by Gel'fond and Linnik in 1949, but they had access to the above formulae only for prime values of $k$ in which case $A_{0}$ is not 0 ; thus they were led to an inequality involving three logarithms of algebraic numbers which they were unable to deal with effectively at that time. By a remarkable coincidence both the formulae for composite $k$ and the desired effective inequality involving three logarithms became available simultaneously in 1966.

Let us now consider the analogous problem for class number 2 . If $Q(\sqrt{ }-d)$ has class number 2 then it is easily seen that $d$ is congruent to 3 or $4(\bmod 8)$, provided $d>15$. Further, when $d \equiv 4(\bmod 8)$ one can immediately write down two inequivalent quadratic forms with discriminant $-d$ in which the coefficients of $x^{2}$ are 1 and 2 respectively, and thus the method described for the class number 1 problem is applicable with only simple modifications (see [5]). There remains the case $d \equiv 3(\bmod 8)$. The theory of genera shows that then $d=p q$, where $p, q$ are primes congruent to 1 and $3(\bmod 4)$ respectively. On signifying by $\chi^{\prime}(n)$ one of the generic characters associated with forms of discriminant $-d$ and writing

$$
\chi_{p q}(n)=\left(\frac{-p q}{n}\right), \quad \chi_{p}(n)=\left(\frac{p}{n}\right), \quad \chi_{q}(n)=\left(\frac{-q}{n}\right), \quad \chi(n)=\left(\frac{k}{n}\right),
$$

where $k$ denotes an integer $\equiv 1(\bmod 4)$ with $(k, p q)=1$, we deduce from classical results of Dirichlet and Kronecker that

$$
\begin{aligned}
L(1, \chi) L\left(1, \chi \chi_{p q}\right) & +L\left(1, \chi \chi_{p}\right) L\left(1, \chi \chi_{q}\right) \\
= & \frac{1}{2} \sum_{F} \sum_{\substack{x=-\infty \\
(x, y) \neq(0,0)}}^{\infty} \sum_{\substack{y=-\infty \\
(x)}}^{\infty}\left(x(F)+\chi \chi^{\prime}(F)\right)(F(x, y))^{-1},
\end{aligned}
$$

where $F$ runs through a pair $f, f^{\prime}$ of inequivalent quadratic forms with discriminant $-d$. We can assume that $f$ is the principal form whence $\chi^{\prime}(f)=1, \chi^{\prime}\left(f^{\prime}\right)=-1$ for all $x, y$. On substituting into the above equa- 
tion and appealing again to Dirichlet's results we obtain

$$
\frac{k \sqrt{ }(p q)}{2 \pi} \sum_{\substack{x=-\infty \\(x, y) \neq(0,0)}}^{\infty} \sum_{\substack{y=-\infty \\ f}}^{\infty} \frac{\chi(f)}{f}=h(k) h(-k p q) \log \epsilon+h(k p) h(-k q) \log \eta,
$$

where $h(l)$ denotes the class number of $Q(\sqrt{ } l)$ and $\epsilon, \eta$ denote the fundamental units in $Q(\sqrt{ } k), Q(\sqrt{ }(k p))$ respectively. Finally, taking $k=21$ and employing similar arguments to those applied in the case of the class number 1 problem we reach the inequality

$$
\left|h(-21 d) \log \epsilon+h(21 p) h(-21 q) \log \eta-\frac{64}{21} \pi \sqrt{ } d\right|<\exp \left(-\frac{1}{10} \sqrt{ } d\right) \text {. }
$$

This has the form

$$
\left|\beta \log \alpha+\beta^{\prime} \log \alpha^{\prime}+\beta^{\prime \prime} \log \alpha^{\prime \prime}\right|<e^{-\delta H},
$$

where the $\beta$ 's denote algebraic numbers with degrees at most 2 , and $\alpha=\eta, \alpha^{\prime}=\epsilon, \alpha^{\prime \prime}=-1, H=\sqrt{ } d, \delta=1 / 10$. Further, the heights of the $\beta$ 's are bounded above by an absolute power of $H$ and the height $A$ of $\alpha$ is bounded above by $p^{\mathrm{c} \vee p}$ for some absolute constant $c$. If $q \leqq d^{1 / 4}$ then the coefficient of $x^{2}$ in $f^{\prime}$ can be taken as $q$ and again the method employed for the resolution of the class number 1 problem is applicable; thus we can assume that $q>d^{1 / 4}$ whence $p \leqq d^{3 / 4}$. It is now clear that to complete the determination of all the imaginary quadratic fields with class number 2 it suffices to establish an estimate $H<C(\log A)^{1+\epsilon}$ for any $\epsilon>0$, where $C=C(\epsilon)$ is effectively computable; and this is precisely the result that Stark [19] and I [6] obtained independently during the summer.

Estimates of the latter kind had occurred previously in connexion with studies concerning the effective solution of Diophantine equations [4], but the arguments used there lead only to an exponent $2+\epsilon$ and not a value $<2$ as required. Our reductions were obtained by somewhat different techniques, Stark's exposition resting heavily on the fact that the $\alpha$ 's are fundamental units in quadratic fields while mine utilized the special condition $\alpha^{\prime \prime}=-1$. Recently we have succeeded in combining our methods and have obtained thereby a general improvement [9]; we have shown in fact that if $\alpha_{1}, \cdots, \alpha_{n}$, $\beta_{1}, \cdots, \beta_{n}$ are nonzero algebraic numbers with degrees at most $d$, if $\alpha_{1}, \cdots, \alpha_{n-1}$ have heights at most $A^{\prime}, \alpha_{n}$ and $\beta_{1}, \cdots, \beta_{n}$ have heights at most $A$ and $B$ respectively and if $\epsilon>0, \delta>0$ and $0<\left|\beta_{1} \log \alpha_{1}+\cdots+\beta_{n} \log \alpha_{n}\right|<e^{-\delta H}$ for some $H>e^{\vee \log B}$ then $H<C(\log A)^{1+\epsilon}$, where $C=C\left(n, d, \epsilon, \delta, A^{\prime}\right)$ is effectively computable. 
And very recently, by further developments of these techniques, there has been established, in the case when the $\beta$ 's are rational integers, a still stronger result incorporating several earlier theorems as special cases (see [7]). This asserts, namely, that if $\alpha_{1}, \cdots, \alpha_{n}$ are nonzero algebraic numbers with degrees at most $d$ and if the heights of $\alpha_{1}, \cdots, \alpha_{n-1}$ and $\alpha_{n}$ are at most $A^{\prime}$ and $A(\geqq 2)$ respectively then, for some effectively computable number $C$ depending only on $n, d$ and $A^{\prime}$, the inequalities

$$
0<\left|b_{1} \log \alpha_{1}+\cdots+b_{n} \log \alpha_{n}\right|<C^{-\log \Lambda \log B}
$$

have no solution in rational integers $b_{1}, \cdots, b_{n}$ with absolute values at most $B(\geqq 2)$. Weaker forms of the assertion have been utilized extensively in the study of number-theoretical problems and most of the results obtained in this respect can now be sharpened. In particular the theorem yields a further effective improvement upon Liouville's inequality of 1844 relating to the approximation of algebraic numbers by rationals; it can in fact be shown that for any algebraic number $\alpha$ with degree $n \geqq 3$ there exist positive effectively computable numbers $c, \kappa$, depending only on $\alpha$, such that

$$
|\alpha-p / q|>c q^{-n+\kappa / \log \log q}
$$

for all rationals $p / q(q>0)$. An improvement of the number on the right to $c q^{-\kappa}$ where $\kappa=\kappa(\alpha)<n$ would follow from the substitution of $\log A \log B$ in the above inequality by $\log A+\epsilon B$ for arbitrary $\epsilon>0$.

Apart from the particular class number questions we have just discussed, the theory of transcendental numbers has also been employed to study several other problems in this field. For instance it has been used by Anfert'eva and Cudakov [1], [2] to make effective certain results of Linnik on the average of the minimum of the norm function over ideals in a given class, and it has further been utilized in some recent joint studies with Schinzel concerning the genera of binary quadratic forms [8]. Furthermore it is, I gather, in the process of being developed to yield results on the class number of quadratic extensions of wider fields than the rationals. Nevertheless the general question of determining all imaginary quadratic fields with a prescribed class number $>2$ remains open. It would seem, however, that transcendental number theory provides, at present, the most promising platform for the launching of further investigations towards the solution of questions of this character and I should expect it to continue to play an important rôle in future researches. 


\section{REFERENCES}

1. E. A. Anfert'eva and N. G. Cudakov, Minima of the norm function in imaginary quadratic fields, Dokl. Akad. Nauk SSSR 183 (1968), 255-256=Soviet Math. Dokl. 9 (1968), 1342-1344. MR 39 \#5472.

2. - Effective lower bounds for the minimum of the norm of.ideals in quadratic fields, Mat. Sb. 82 (1970), 55-66 = Math. USSR Sb. 11 (1970), 47-58.

3. A. Baker, Linear forms in the logarithms of algebraic numbers. I, II, III, IV, Mathematika 13 (1966), 204-216; ibid. 14 (1967), 102-107, 220-228; ibid. 15 (1968), 204-216. MR 36 \#3732.

4. - Contributions to the theory of Diophantine equations. I. On the representation of integers by binary forms; II. The Diophantine equation $y^{2}=x^{3}+k$, Philos. Trans. Roy. Soc. London Ser. A 263 (1967/68), 173-208. MR 37 \#4005; 4006.

5. - A remark on the class number of quadratic fields, Bull. London Math. Soc. 1 (1969), 98-102. MR 39 \#2723.

6. - Imaginary quadratic fields with class number 2, Ann. of Math. (to appear).

7. - - A sharpening of the bounds for linear forms in logarithms, Acta Arith. (to appear).

8. A. Baker and A. Schinzel, On the least integers represented by the genera of binary quadraticforms, Acta Arith. (to appear).

9. A. Baker and H. Stark, On a fundamental inequality in number theory, Ann. of Math. (to appear).

10. R. Brauer, On the zeta-functions of algebraic number fields, Amer. J. Math. 69 (1947), 243-250. MR 8, 567.

11. A. O. Gel'fond, Transcendental and algebraic numbers, GITTL, Moscow, 1952; English transl., Dover, New York, 1960. MR 15, 292; MR 22 \#2598.

12. A. O. Gel'fond and Yu. V. Linnik, On Thue's method in the problem of effectiveness in quadratic fields, Dokl. Akad. Nauk SSSR 61 (1948), 773-776. (Russian) MR 10,354 .

13. K. Heegner, Diophantische Analysis und Modulfunktionen, Math. Z. 56 (1952), 227-253. MR 14, 725.

14. H. Heilbronn, On the class number in imaginary quadratic fields, Quart. J. Math. Oxford Ser. 5 (1934), 150-160.

15. H. Heilbronn and E. H. Linfoot, On the imaginary quadratic corpora of class number one, Quart. J. Math. Oxford Ser. 5 (1934), 293-301.

16. C. L. Siegel, Über die Classenzahl quadratischer Zahlkorper, Acta Arith. 1 (1935), 83-86; Ges. Abhandlungen I, 406-409.

17. H. M. Stark, On complex quadratic fields with class-number equal to one, Trans. Amer. Math. Soc. 122 (1966), 112-119.

18. —, A complete determination of the complex quadratic fields of class-number one, Michigan Math. J. 14 (1967), 1-27. MR 36 \#5102.

19. —_ A transcendence theorem for class-number problems, Ann. of Math. (to appear).

Trinity College, Cambridge, England 